\title{
Structural realism versus deployment realism: A comparative evaluation
}

\author{
Timothy D. Lyons \\ Department of Philosophy, Indiana University-Purdue University Indianapolis, Cavanaugh Hall, 425 University Blvd., Indianapolis, IN 46202, USA
}

\section{A R T I C L E I N F O}

\section{Article history:}

Received 29 June 2016

Available online 22 July 2016

\section{Keywords:}

Scientific realism

Structural realism

Deployment realism

Pessimistic induction

Divide et impera

Novel predictions

No-miracles argument

\begin{abstract}
A B S T R A C T
In this paper I challenge and adjudicate between the two positions that have come to prominence in the scientific realism debate: deployment realism and structural realism. I discuss a set of cases from the history of celestial mechanics, including some of the most important successes in the history of science. To the surprise of the deployment realist, these are novel predictive successes toward which theoretical constituents that are now seen to be patently false were genuinely deployed. Exploring the implications for structural realism, I show that the need to accommodate these cases forces our notion of "structure" toward a dramatic depletion of logical content, threatening to render it explanatorily vacuous: the better structuralism fares against these historical examples, in terms of retention, the worse it fares in content and explanatory strength. I conclude by considering recent restrictions that serve to make "structure" more specific. I show however that these refinements will not suffice: the better structuralism fares in specificity and explanatory strength, the worse it fares against history. In light of these case studies, both deployment realism and structural realism are significantly threatened by the very historical challenge they were introduced to answer.
\end{abstract}

(ㄷ) 2016 Elsevier Ltd. All rights reserved.

\section{Introduction: deployment realism and structuralism}

This paper endeavors, ultimately, to examine structural realism as an explanation for novel predictive success. A great lesson of 20th century philosophy of science is that evaluations of empirical hypotheses are comparative and ultimately triadic: it is not merely hypothesis versus data; it is rather hypothesis versus hypothesis, with adjudication between them being focused in part on data. The relevant data in my empirically triadic evaluation are those from the history of science. And the foil against which I will compare structural realism is the more robust, and arguably the most sophisticated, variant of scientific realism, deployment realism. Before turning in the spirit of Laudan (1981) to the historical data, we can compare these positions conceptually.

According to deployment realism, we can be justified in believing the following meta-hypothesis: those theoretical constituents that were genuinely deployed in the derivation of novel predictive success are at least approximately true. The justification for believing this meta-hypothesis: it would be a miracle were our deployed theoretical posits to achieve such successes were they not at least approximately true. Deployment realism has much going

E-mail address: tdlyons@iupui.edu. for it. It is testable, as I will try to make clear below. It is applicable, offering the promise of allowing us to identify, in at least some instances, those constituents to which we can, according to deployment realism, commit ourselves. And it appears to be genuinely explanatory: credit for success is not due to those elements that were mere "idle wheels," rather it is due to those and only those that were responsible for the particular successful predictions. While the need for comparative evaluation is a central lesson from 20th century philosophy of science in general, this emphasis on the proper attribution of credit may be one of the central additional lessons arising from the realism debate, in particular. Forgetting either lesson would constitute a backward step.

Worrall looking to Poincare embraces what he calls "syntactic or structural realism" (1989, 157; see also 152), singlehandedly introducing it to the contemporary scientific realism debate. Structural realism (hereafter "structuralism") purports to be a logically weaker, less committal, position than deployment realism: we can be justified in, and need only commit ourselves to, believing the structures expressed by those constituents genuinely deployed toward success. Like deployment realism, structural realism appeals to the no-miracles argument articulated above, but suitably modified: the only explanation for novel success is that the structural elements of those constituents responsible for those successes 
capture the actual structure of the world. Comparative plausibility requires that structuralism meets the demands met by deployment realism. It must be testable. The structural elements of the deployed constituents must be identifiable. And, in order to stand as a genuine explanation of novel predictive successes, the structure must be genuinely deployed toward the novel predictions. Beyond embracing the same explanatory argument, structuralism shares with deployment realism another feature: both Worrall and Poincare brought it in to deal with the history of science, in particular, instances in which historical theories were successful but are nevertheless now taken to be false. Although we cannot say that Fresnel's ether theory is approximately true, it was impressively successful, predicting, for instance, the infamous white spot in the center of the shadow of an opaque disk. How are we to make sense of this success, given the rejection of Fresnel's ether theory by contemporary science? Worrall answers, "there was continuity or accumulation in the shift, but the continuity is one of form or structure, not of content" (157). Because structuralism is less demanding, we should expect that, with respect to given instances of novel successes, it fares better than, or at least as well as, deployment realism. Offering an explanation for novel predictive success, along with an ability to handle the historical data that challenges traditional realism, structuralism purports to offer "the best of both worlds," to use Worrall's apt phrase (1989).

The great question left by Worrall's hint is, How precisely are we to understand structure? It turns out that structuralism has a long history. And, drawing on various early 20th century philosophers, it has come to take on many forms. One might well claim that there are more variants of structuralism than there are structuralists, since a number of individual structuralists, Worrall among them, have changed their positions. I anticipate that my historical considerations below will not only pose threats to deployment realism but will at least require clarification of some of forms of structuralism, if not significant revision. The latter result, of course, would invite even more variants.

Much discussion of structuralism has focused on a few sets of historical theories: the successes of 19th century ether theories, e.g. Fresnel's, and quantum mechanics, e.g. on the question of whether, in light of that theory, the notions of individuality, objecthood, intrinsic properties, etc, can be retained. Building on my (Lyons, 2006), the focus in this paper will be on the history of other cases from the well-known arena of celestial mechanics, in which both deployment realists and structuralists have tended to think their position fares unquestionably well: I will start with a few comments on Kepler, Newton, Leverrier and Adams, then move to predictions made by general relativity. Examining arenas of theory change that appear, initially, non-threatening to both the deployment realist and the structuralist, and then showing that those arenas do pose a threat, reveals that threat to be especially pressing.

Since deployment realism and structuralism share those core components captured above, my approach will be as follows: I will first discuss historical threats to deployment realism and then, in due course, ask whether structuralism promises to do as well or better-or whether, by contrast, the structuralist is in just as bad or (surprisingly, perhaps) worse shape given the particular historical cases brought forward. We will see that, leveraged by those cases, the structuralist's need for the retention of structure compels the structuralist toward increasingly vacuous conceptions of "structure." The better structuralism fares against the historical examples, the worse it fares in content and explanatory strength. In light of that problem of explanatory vacuity, the structuralist will be compelled to embrace some explicitly restrictive notions of structure, against which, in Section 4, I will direct the cumulative force of the discussion. The better structuralism fares in specificity and explanatory strength, the worse it fares against history. The upshot: structuralists are trapped by their need for historical retention of structure and their own explanatory demand.

\section{Some challenges from the history of celestial mechanics}

\subsection{Preliminary cases}

In his (1596) Mysterium Cosmographicum, Kepler articulated his theory of the anima motrix, a theory that causally explained some primary features of planetary motion. Because that text was written years before Kepler met Brahe, it is clear that it was not put forward to accommodate Brahe's detailed data. And what we find is that, directly deploying its foundational posits, now taken to be patently false, Kepler made a series of temporally novel predictions. Toward the latter, seeking to explain why the planets are moved in paths around the sun, he posited the following: planets move only when forced to move; they could not move themselves, because they would tire; the sun is unique and in fact supreme, not by its size, but its divinity; it is positioned at the center of the universe; the sun is that which pushes the planets in their orbits; it emits rays that do the pushing, the anima motrix, etc. Later, in a 1605 work, Astronomia Nova (1609), Kepler derived from that theory the prediction that the sun spins: "since the [emanation] of the source, or the power moving the planets, rotates about the center of the world, I conclude with good reason... that that of which it is the species, the sun, also rotates" (1609, p. 387 ). He also predicted that the sun spins in the direction of planetary motion and that it spins along the plane of the ecliptic. Returning now to his (1596), beyond providing a physical explanation of why planets moved, he wanted to explain, why those with a greater mean distance from the sun traverse their orbits at a slower pace than those closer to the sun. To do this, he conjoined to the above posits the hypothesis that, as the anima motrix pushes the planets, its strength decreases in proportion to their mean distances from the sun: "there is a single moving soul in the center of all the spheres, that is, in the Sun, and it impels each body more strongly in proportion to how near it is. In the more distant ones on account of their remoteness and the weakening of its power, it becomes faint, so to speak" $(1596,199)$. In answering how that force diminishes, he invoked his hypothesis that the intensity of light is inversely related to distance from the sun. Moreover, "motion is dispensed by the Sun in the same proportion as light" $(1596,201)$. From these, in conjunction with the posits above, he arrived at his prediction that the sun spins faster than any of the planets revolve around it (1609, 387-8). Further, the anima motrix gave him "the reason and the means" to "defend" the "irregularity in" the planetary paths: a "planet will be slower" when "further away from the Sun," where it is "moved by a weaker power," and "faster" when "closer to the Sun," where it is "subject to a stronger power" $(1596,217)$. This constituted the novel predictions that planetary motion is non-uniform and, more specifically, that each planet will reach its highest speeds at its perihelion and its lowest at its aphelion. Brahe's data confirmed this for Mars, Jupiter, and Saturn, rendering the predictions at least use-novel, and eventually for Mercury, Venus, and the Earth, instances of temporally novel success. These predictions would later be accepted as holding for the planets unknown to Kepler, Uranus and Neptune. Although some of his predictions may have been articulated later, the posits I've flagged as pivotally deployed toward them predate his access to Brahe's data.

In his (1609), directing his theory of the anima motrix toward what came to be known as his second law, Kepler supplemented his reasoning with the posit that the planet's own inclination to be at rest takes over as the sun's push diminishes $(1609,384)$. He also looked to Gilbert's theory of magnetism, reasserting, however, that 
the anima motrix is "only a directing force," explicitly denying any attractive component: "there is in the sun no force whatever attracting the planets, as there is in the magnet" $(1609,390)$. Each of these constituents of Kepler's deep theory were centrally deployed in arriving at his "laws," which were themselves central to the unprecedented success of the Rudolphine Tables. Those laws led to, and continue to lead to, innumerable successful predictions pertaining to the behavior of, not only Mars and the Earth, but also Mercury, Venus, Saturn, and Jupiter. Early on, Kepler achieved additional temporally novel successes predicting specific relations between the Earth, Sun, and planets: he predicted, not only two planetary transits-the Mercurial, and the rare and irregular Venusian, transit-but also a short separation between the two transits. And his laws led to, and continue to lead to, a multitude of novel successful predictions regarding the then undiscovered planets, Uranus and Neptune, the dwarf planets, and legions of other bodies in the solar system and beyond. The reasoning Kepler employed in arriving at these novel predictions centrally included each of the patently false components of Kepler's deep theory identified above.

Beyond the predictions derived by Kepler himself, the deployment realist's criterion requires that we credit Kepler's laws, obtained via the anima motrix, with other predictive successes. For instance, they were utterly fundamental to Newton's De motu corporum in gyrum (1684), the pre-Principia text in which Newton arrives at his revolutionary conclusion that planets are subject to an inverse-square instantaneous action-at-a-distance gravitational attraction to the sun. Newton posits that innate forces push masses, which are otherwise unmoved, in a straight line infinitely (Hypothesis $2,1684,1)$. Asserting this to obtain for a single point-mass in the universe, which moves in an ellipse, and deploying Kepler's second law, he shows that the required force toward a focus of the ellipse would be inversely proportional to the distance squared (Problem 3, following Theorem 3, 1684, 5). This is the fundamental instance from which Newton generalized to arrive at his law of universal gravitation. Newton required of that distance-force relation that it accord with Kepler's laws, and its generalization constituted the foundation for his law of universal gravitation: The instantaneous action-at-a-distance gravitational force between two objects is proportional to the product of their masses and inversely proportional to the square of the distance between them. In my (Lyons, 2006) I show that, by cumulative force, a set of at least ten false Keplerian constituents must be credited with the novel Newtonian successes identified below, and that to deny this would sacrifice the content of deployment realism, leaving it arbitrarily contrived.

However, notably, that is not the only point to be drawn from our discussion of Newton. Newton deployed his own false posits that were by no means idle wheels in the success of Newtonian mechanics-e.g., a point mass is propelled forward by an innate force; it is also pulled by an inverse-square force toward a focus of its elliptical path; this constitutes the entire content and behavior of the universe. Along with the Keplerian foundation, and by way of Newton's law of universal gravitation, the deployment realist must credit these-irrespective of how quickly they are contradicted-with a set of predictions that were at least use-novel, including the slowing of Saturn as it passed Jupiter, the behavior of the tides, the behavior of stellar objects, and the precession of the equinoxes. Nor can these be denied a central role toward the successful temporally novel Newtonian predictions pertaining to, for instance, the oblate shape of the earth, the returns of Halley's comet, many nonKeplerian perturbations, as well as any number of recent predictions, such as the hurling of Apollo 13 around the moon and other gravity assists such as those achieved in respect to Voyagers 1 and 2 , etc.
Soon after William Herschel discovered Uranus in 1781, it was realized that its observed behavior failed to accord with predictions. In an extra-ordinary effort to causally account for the discrepancies, both Leverrier and Adams successfully predicted the existence of a trans-Uranian planet, never previously observed as such. Moreover they made exceptionally accurate specifications of Neptune's position in the vast Autumn sky of 1846. John Herschel reporting Galle's observation, wrote "The geo-centric longitude determined by $\mathrm{Dr}$ Galle from his observation was $325^{\circ} 53^{\prime}$, which, converted into heliocentric, gives $326^{\circ} 52^{\prime}$ arc minutes, differing $0^{\circ} 52^{\prime}$ from M. Leverrier's place, $2^{\circ} 27^{\prime}$ from that of Mr Adams, and only $47^{\prime}$ from a mean of the two calculations" $(1849,668)$. Most significantly for our concerns, each, working independently of the other, deployed numerous false constituents toward their successful predictions. For instance, the constituents each deployed regarding the planet's semi-major axis greatly exceeded today's value: Leverrier was off by six times the earth's distance from the sun (36.1539 AU); Adams by seven times (37.2474 AU). Related to that, each deployed false constitutents regarding the distance between the semi-major axes of Neptune and of Uranus, Leverrier being off by nearly 17 times and Adams's more than 18 times the earth's distance to the sun. Regarding Neptune's mass, Leverrier's value was more than double today's, while Adams's value was nearly triple, the former off by over 18 times the earth's mass, the latter overreaching by nearly 33 times that mass. Furthermore, for both, the orbit posited for Neptune was far too eccentric: Leverrier exceeding 12.5 times (0.10761), and Adams 14 times (0.120615), the present measure (0.00859).

Beyond, but related to, these central constituents, numerous other false constituents were involved. For instance, for both systems, the orbital periods overshot Neptune's current value by more than half an earth century. And the constituent regarding the longitude of the perihelion was dramatically false $-284^{\circ}, 45^{\prime}$ for Leverrier; and $299^{\circ}, 11^{\prime}$ for Adams, compared with a present value of $44^{\circ}, 58^{\prime}$. Finally, the predictions relied on any number of additional false constituents pertaining to Uranus itself, for instance its mass, orbital period, eccentricity, etc. (Herschel, 1849, 669). Two of the most astonishing temporally novel successes to be referenced in the history of science-the existence of a trans-Uranian planet and its location as a point of light in the Earth's vast sky-depended on the large collection of false posits identified above.

\subsection{Light, gravity, space, and time}

Considering the great novel predictive successes of celestial mechanics, we naturally step toward the successes of general relativity. Einstein wrote in (1916c): "Apart from" the prediction regarding mercury "it has hitherto been possible to make only two deductions from the theory which admit of being tested by observation, to wit, the curvature of light rays by the gravitational field of the sun, and a displacement of the spectral lines of light reaching us from large stars" (1916c, 123-4). Surprisingly, it turns out that the latter prediction was made more than a century and a quarter earlier, along with others generally attributed solely to general relativity made in the same time period.

In 1784 John Michell predicted the spectral shift of light from massive stars. Among his deployed constituents were the following: light is not wavelike; it is made up of corpuscles; light corpuscles have mass; light corpuscles follow the same laws as matter; the speed of light varies; gravity affects "the velocity of light" corpuscles emitted from a body (1784, 37); a luminous massive object will exert a pull on the light corpuscles it emits, the latter being "attracted in the same manner as all other bodies with which we are acquainted" $(1784,37)$; the velocity of the light corpuscles will be decreased in proportion to the mass of the luminous 
object; that velocity will determine the way in which those light corpuscles interact with an optical medium on a second body, e.g., the earth; velocity variation will bear on light refraction, spectral shifts revealing a difference in the velocity of light corpuscles. Since a prism provides a way to determine a difference in refraction, it can inform us of a difference in the velocity at which light corpuscles are moving. With this line of reasoning we arrive at a novel prediction, decades even before Fraunhofer invented the spectroscope in 1814: we can obtain information about the properties, e.g., the mass of distant stars by measuring "the refrangibility of the light" (51). A second novel prediction is that a star with a large mass will bring about a spectral shift when compared to one of lesser mass. Related to this, we arrive at the prediction that we can compare the masses of the stars in binary star systems "systems of bodies revolving about each other" (56) by looking for a shift in spectra.

Michell made another 1784 prediction regarding the possibility of massive dark bodies. Deployed along with those constituents just noted, e.g., the velocity of the light corpuscles will decrease in proportion to the mass of the stellar object, Michell reasoned, if that object is sufficiently massive that its gravitational force overtakes the velocity of the light corpuscles, the light corpuscles will not escape: "all light emitted from" a sufficiently dense "body would be made to return towards it, by its own proper gravity" (42). With this we arrive at the prediction that stellar objects of a sufficient mass will result in invisible dark stars. Moreover, their existence would be indicated by other bodies revolving around them: "If there should really exist in nature any bodies, whose... light could not arrive at us... we could have no information from sight; yet, if any other luminous bodies should happen to revolve about them we might... infer the existence of the central ones" (Michell to Cavendish, 1783, qtd in Glendenning, 2007, 176; see also Michell, 1784, 50). In 1796 Laplace likewise made this prediction regarding dark bodies and, even beyond that, arrived precisely at what would later be called the Schwarzschild radius: Glendenning writes, the "critical radius" of Laplace's Dark Body, "is $\mathrm{R}=2 \mathrm{GM} / \mathrm{c}^{2}$, which is the Schwarzschild radius of a black hole! (It is not clear to this [author] why classical reasoning should have led to an exact result of General Relativity.)" (2007, 176).

General relativity's prediction of the deflection of light in conjunction with its confirmation by the 1919 Eddington expedition may well constitute the exemplary instance of novel predictive success. However-otherwise unacknowledged in the realism literature, as far as I can tell-it turns out that this impressive prediction had been made by Johann Georg von Soldner in 1801, more than a century before the Eddington expedition was conceived. In fact, beyond being anticipated by Cavendish in 1784, Newton himself alluded to such a possibility in 1704: with "yes" as the implied answer, he asked, "Do not bodies act upon light at a distance, and by their action bend its rays, and is not this action (ceteris paribus) strongest at the least distance?" $(1704,132)$. The prediction itself is explicit in the title of von Soldner's text: "On the deviation of a light ray from its motion along a straight line through the attraction of a celestial body which it passes close by." The theoretical constituents von Soldner deployed include the following: light is not wavelike; it is made up of corpuscles; light corpuscles have mass: "I treat a light ray as a heavy body" (von Soldner, 1801, 948); light follows the same laws as matter: "Light rays have all the absolute [basic] properties of matter" (translator's brackets, von Soldner, 1801, 948); the speed of light varies; an instantaneous gravitational force affects the velocity of light corpuscles; more specifically, that force attracts light corpuscles: "The light ray will... be forced [to curve], because of the attraction of the celestial body" (von Soldner, 1801, 940). His prediction is that light will bend around massive objects, and his more specific prediction is that it will bend at " 0.84 " seconds of arc (1801, 947; see Jaki, 1978.)

In advance of the Eddington expedition, Heber Curtis and William Wallace Campbell, the head of the Lick observatory, conducted eclipse measurements in 1918. During the 11 July 1919 meeting of the Royal Astronomical Society, Campbell reported: "It is my opinion that Dr. Curtis's results preclude the larger Einstein effect but not the smaller amount expected according to the original Einstein hypothesis" (1919, 299), that smaller amount approximating von Soldner's result. Although the results of that 1918 expedition were never published, Curtis wrote to Campbell: "My own strong preference, however, would be to append... a brief description of the plates, the measurements and the results." He offered the following: "The simple mean of the measurements made on the Goldendale plates in both coordinates and of six plates of the 1900 eclipse in declination is $0 .{ }^{\prime \prime} 69 \pm 0$." 02 for the deflection at the Sun's limb. When weighted in accordance with the values assigned in the following short table, the value of the deflection at the Sun's limb becomes $0 . " 74 \pm 0$ " .03 ." After presenting the table he writes, "the mean of the above results suggests the gravitational deflection of 0.87 at the Sun's limb." (Curtis to Campbell, 29, 1920, Dec; qtd in Earman \& Glymour, 1980, 65-66). According to Earman and Glymour, this is "the closest thing we have to a report of the 1918 expedition" (65). Later Eddington oversaw a six month expedition to Sobral, Brazil, and the island, Principe, near Africa. The Sobral experiments, reported by Dyson, were conducted on a clear and sunny day, while Eddington's own measurements were made in Principe, where it was cloudy. Eighteen of the Sobral astrograph telescope plates gave an average of 0.86 seconds of arc (Earman \& Glymour, 1980, 74). The results “confirmed [Einstein's] theory only if part of the observations were thrown out and the discrepancies in the remainder ignored. Dyson and Eddington, who presented the results to the scientific world, threw out a good part of the data and ignored the discrepancies" (Earman \& Glymour, 1980, 85). "The blurred and dumbell-shaped images" of the only "two usable plates" from Principe "were worst of all" (74) but gave on Eddington's analysis an average of 1.61 seconds of arc (75). The 18 astrographic plates from the Sobral expedition were discarded due to the clear sky, determined in retrospect to be distorted by the sun's heat.

While on the topic of Einstein's three central predictions-having added "dark bodies" for good measure-we should note "The closing of a famous astronomical problem," to use the title of a (1909) article written by Campbell. The problem is the advancement of Mercury's perihelion. And since Einstein's general relativity was then unarticulated, the reader properly anticipates that this is no a reference to that theory. Rather, it is a fully Newtonian solution. The proposal was that the advancement of Mercury's perihelion is due to collection of individually invisible planetoids. That postulate also predicted, in the use-novel sense, instances of the glow in the sky's horizon, before sunrise in the east and sunset in the west. Simon Newcomb wrote, we can "regard it as certain that, if a group of intra-Mercurial planets exists, its members are too small to be seen when projected on the sun's disk..." (1911, 155). He wrote, "if the group exists the members must be so small as to be [individually] entirely invisible. But in this case they must be so numerous that they should be visible [collectively] as a diffused illumination on the sky after sunset. Such an illumination is shown by the zodiacal light." (Newcomb, 1910-1911, 155; quoted with brackets in Grünbaum, 1976, 356). Newcomb points to a version of Hugo von Seeliger's (1896) hypothesis: "In 1896 H. H. Seeliger made a more thorough investigation than his predecessor [Newcomb himself] had done of the attraction of the matter producing the zodiacal light, assuming it to be formed of a series of ellipsoids. He showed that 
the motions of the nodes and perihelion could be satisfactorily represented in this way" $(1911,155)$. Newcomb accepted von Seeliger's as the best hypothesis to date, "the most probable one in the present state of knowledge" $(1911,155)$. That is also the hypothesis that, in Campbell's (1909) article, closed the "famous astronomical problem."

\section{Implications for structuralism: historical pressure toward a problem of explanatory vacuity}

\subsection{The structure of light}

We have now introduced a set of novel predictions that are included among the most significant in the history of science, and we see that, toward each, sets of patently false theoretical constituents were deployed. As implied, I take the preceding exploration to pose serious historical challenges to deployment realism. We can now focus on structuralism. Above, we saw that, since only those components that are genuinely deployed toward novel success can explain novel success, structure must be included in what is genuinely deployed toward success. ${ }^{1}$ Generally, both structuralists and their critics take for granted that the virtues of structuralism are particularly apparent in the context of theorizing about light, an arena of theorizing thus rendered ideal for identifying challenges. We saw Kepler posit that sunlight diminishes inversely with the distance it travels and very explicitly deploy this postulate toward his determination of the changing strength of the sun's push. Both were posits of inverse rather than inverse-square relations, and both were deployed toward a set of novel predictions flagged above-for instance, regarding the apsidal distance-speed relations of planets, and toward Kepler's incredibly fruitful second law. Now take Michell's prediction regarding the spectral shift of light from massive bodies, as well as his prediction of the possibility of dark bodies, Laplace's determination of the Schwarzschild radius, and, finally, von Soldner's prediction of light bending around massive objects. Each of the previously articulated theoretical constituents pertaining to light, among the entire set of constituents identified above, were deployed toward one or all of these novel successes, and each is patently, and I will suggest, structurally, false.

Scientific realists want to claim, minimally, not only that novel successes of a successor system reveal a greater approximation to truth than its predecessor system but that, as science advances toward the truth, newer theories will fare better than their predecessors on specific successful novel predictions. Moreover, comparing Newtonian corpuscular theory against Maxwell's later wave theory, realists are compelled to take the latter as a significant advance over the former toward truth. However, given that a massless wave is wholly unaffected by Newtonian gravity, each of these novel predictions, along with each corpuscularian constituent responsible for it, is lost for a century. Quite to the contrary of what realists expect, replacing falsity with what realist's must consider a greater approximation of theoretical truth results in a century of loss of novel (and later successful) predictions. Nor, however, can we say that those lost false theoretical posits responsible for those novel successes express the real structure of light. According to contemporary science, it is patently not the case that, structurally, light obeys the same laws as matter; permits of variability in its speed; can have a vacuum speed so slow as to be overcome by the speed of gravitational attraction; possesses what

\footnotetext{
${ }^{1}$ Moreover, because structuralists invoke the no-miracles argument and reject non-structural content, for structuralists, as Psillos $(1999,153)$ points out, it is only the structural content that can be genuinely deployed toward success.
}

structurally amounts to mass; undergoes an instantaneous actionat-a-distance attraction toward matter in an inverse-square relation to distance, etc. We have numerous theoretical constituents pertaining very specifically to light that were deployed toward a set of successful novel predictions but which cannot be said to accurately express or even approximate light's structure. This appears to be a direct strike at the very context in which structuralism was brought into the scientific realism debate, that of theorizing about light, the context in which structuralism was introduced and is taken to thrive.

\subsection{The structural relation between light, gravitation, mass, distance, spacetime...}

Of course, to allow for all of these false structural posits regarding light, and needing to nonetheless reassert that structure has been retained, our structuralist will now be prompted to modify the meaning of "structure," exploiting the fluidity of that key term. ${ }^{2}$ No longer able to explain the novel successes by a conception of structure that pertains to light itself, our structuralist may well hope those successes can be explained by the claim that the relevant constituents properly capture the structural relation between light, gravitation, mass, distance, spacetime, etc. For instance, our structuralist might suggest that "light follows the same laws as matter" structurally approximates the contemporary specification that "light, no less than matter, follows the warping of spacetime."

Addressing this prima facie promising response requires attending to the nature of the shift from Newtonian to relativistic structure. Notably, as with explicit discussions restricted to the nature of light, we again enter an arena taken to exhibit the advantages of structuralism. For instance, though Worrall emphasizes radical content change between the Newtonian and relativistic conceptions (1989, 143 and 148), he introduces his structuralism as the best of both worlds, purporting to permit that shift while also capturing the no-miracles intuition. Our present query is whether that shift also involved radical structural change.

Newton writes, while "the common people [or "the vulgur"] conceive" such "quantities" as space and time "under no other notions but from the relation they bear to sensible objects" (6), beneath these relative notions lie the absolutes, the permanent and immovable. Specific "times and spaces are, as it were, the places as well of themselves as of all other things" $(1687,8)$. The "parts of space" are ordered immutably (8). Space depends on nothing else, is "of its own nature" and non-relational, "without reference to anything external." It "always remains similar," is entirely homogeneous. Space "always remains... immovable." It is permanent as it is, and there is nothing into which it could move (6). Time is likewise absolute, "of itself," subsisting "without regard to anything external," unaffected and independently flowing "from its own nature" (6), its "parts" ordered so as to be "immutable" (8). Structurally, both space and time are infinite, independent of one another, and independent of everything else, for instance, light. Events occur in definite immutable "parts" of space and time. As for motion and rest: "Absolute motion is the translation of a body from one absolute place into another" (7); and "real, absolute rest, is the continuance of the body in the same part of that immovable space" (7). Within this absolute world structure, light, mass, and distance

\footnotetext{
${ }^{2}$ Although, to give the structuralist the benefit of the doubt against the case studies, I will follow the structuralist in this retreat from specificity, in Section 4, I will direct the cumulative force of my discussion against the key restrictions on "structure" that structuralists have recently imposed.
} 
are unchanging, and gravitation occurs instantaneously by way of an action-at-a-distance force.

With the (temporary) exception of the gravitational force, special relativity denies each such property of that metric structure. Space and time become deeply entwined, their relation being inextricably related to light; mass becomes mutually convertible with energy, so not itself conserved, and as with distance, is altered according to its relation to light and non-absolute frames of reference. All of this stems from Einstein's revolutionary posit that the speed of light is invariant irrespective of reference frames. It is precisely on the reconceptualization of light that the relativistic revolution against Newtonianism pivots. That is the difference that makes all the difference. Though we may well all realize this when pressed, I know of no other such acknowledgement in the structuralism literature on light. General relativity ushers in even more dramatic structural changes, its pivot point being the denial of gravitation as an instantaneous action-at-a-distance force. While special relativity may retain a global invariance of coordinate transformations, with general relativity even that global structure is lost: transformations become entirely local. Granted, at a given "event," provided we restrict ourselves to a local "patch," away from, say, a black hole, spacetime may be Minkowskian; however, beyond local patches, information about spacetime structure at least appears to require information about stress-energymomentum. Spacetime has, not only a variable structure, but a seemingly dependent structure. And it is not merely that what happens in spacetime is structurally dynamic, spacetime itself is structurally dynamic. This constitutes a dramatic upheaval of Newtonian structure, appropriately dubbed revolutionary. Moreover, those constituents on which this structural revolution pivots are, I submit, the denial that the speed of light varies and the denial that gravitation results from an instantaneous action-at-a-distance force.

These points in hand, and returning to the specifics of our case studies, we saw that Michell, Laplace, and von Soldner undeniably included, for instance, spatial postulates in their reasoning. However, these have nothing to do with the structural curvature we would now say obtains around a large mass. So even if "space is absolute" was not genuinely deployed toward those Newtonian successes, those successes are clearly not due to any posit regarding the structure of spacetime as now understood. Moreover, we saw that these Newtonians (Laplace aside) very explicitly deployed the variability of the velocity of light, and in particular, its slowing. Claiming that this argumentatively pivotal variation in $c$ somehow structurally approximates the absolute invariance of $c$ depletes the content of an indisputably central structural component of our current conception. Further, we've seen that the invariance of $c$ has revolutionary implications for classical space and time-e.g., converging the two into a single spacetime that is sliced in innumerably many ways, and "in" which mass/energy and distance vary according to coordinate frames. We've also seen that those Newtonian predictions depend on the slowing of light corpuscles that are overcome by the instantaneous gravitational force. Combining these points, those Newtonian successes depend on, not only the absolute separation between space and time, mass and distance, so space and mass, mass and energy, etc. but on each being individually absolute at least as contrasted against the relativistic conception, for shorthand, let's say, contrastively absolute (as opposed to "absolute in every Newtonian detail"), where, irrespective of coordinate frames, it is determinedly not the case that time can be dilated, spatial length can be contracted, and so on. The structuralist now appears forced to claim that contrastively absolute space and contrastively absolute time, along with invariant mass, energy, distance, etc., structurally approximate the extraordinary relativity of spacetime slicing, the variance of mass, of energy, of distance, etc.
However, such claims of structural approximation are at least as problematic as claiming the significant variance of light's velocity structurally approximates light's absolute invariance. I proposed above that those constituents that launch the structural revolution are, with special relativity, the invariance of light irrespective of coordinate systems and, with general relativity, the elimination of a non-instantaneous gravitational force. To this we can add that, in our contemporary system, what renders patently false the constituents responsible for the Newtonian successes of Michell, Laplace, and von Soldner are precisely those pivotal constituents of the structural revolution. These points conjoined, that which makes all the structural difference between the Newtonian and relativistic systems is the difference between, on the one hand, the light invariance along with force elimination constituents and, on the other, the Newtonian constituents deployed toward novel success. Not only are the resulting differences of the revolution structurally relational (as above, e.g., independent space and independent time versus structurally dynamic spacetime), so are the constituent-sets that fundamentally differentiate the two systems. We have, then, a structural relational revolution-two relational constituent-sets, one Newtonian the other relativistic, that render one another patently false.

We've seen that the grand question arising in the context of structuralism is, just what is it that we can justifiably believe our theories have got right? I've argued that, by present theory, we can claim neither that the Newtonians had the structure of light right, nor that they had the correct structural relation between light, gravitation, mass, distance, spacetime, etc. I am applying the historical cases as pressure against the notion of structure, forcing a notion of structure that is increasingly vacuous. This will be an important line in what follows.

\subsection{Equations that express structural relations}

Having now addressed the structural disparity between Newtonian and relativistic conceptions of the world, we are pushed to address the question of just how general relativity accords with structuralism, even setting aside those historical relations. Toward that end, and an understanding of "structure," it is noteworthy that (more often than not, I dare say), the structuralist appears to equate structure with a theory's equations: "if we restrict ourselves to the level of mathematical equations-not notice the phenomenal level- there is in fact complete continuity between Fresnel's and Maxwell's theories" (Worrall, 1989, p. 158). Worrall explicitly draws on Poincare who writes, "these equations express relations, and if the equations remain true, it is because the relations preserve their reality. They teach us now, as they did then, that there is such and such a relation between this thing and that... The true relations between these real objects are the only reality we can attain" (1905, qtd in Worrall, 1989, 158). Outside of the Fresnel case, Worrall writes, "On the structural realist view, what Newton really discovered are the relationships between phenomena expressed in the mathematical equations of his theory..." $(1989,162)$. So here at least descriptions of relations, expressed in mathematical equations, are what we can justifiably believe and are what are retained across theory change. In the first sentence of Worrall's launch of structuralism into the scientific realism debate, the first in his list of "presently accepted physical theories" is "a curved space-time structure" $(1989,139)$. He writes, "Most of us unreflectingly take it that... those theories really do straightforwardly assert that space-time is curved in the presence of matter" $(1989,139)$. When detailing the justification for realism, the no-miracles argument, Worrall includes general relativity, in particular, as making so "many correct empirical predictions" (1989, 140; see also 2011, 158). Moreover, general relativity is, fundamentally, a single 
mathematical equation: in condensed form, it is $G_{\mu \nu}=8 \pi T_{\mu \nu}{ }^{3}$ Additionally, the $8 \pi$ is written into it to preserve, that is deliberately accommodate, to some degree, an empirical core of the Newtonian framework. Moreover, between the quantities on each side of " = " that equation clearly expresses relations. Finally the equation is directly tied to spacetime structure. Hence, looking to identify a structuralist commitment, we appear to be on track. (And mindful of both the need to explain success and the extraordinary role general relativity plays in our current conception of reality, structuralists who have been content to leave it to the side are compelled to clarify just what in that theory their principles commit them to.) However, consideration of the Einstein equation itself reinforces our recognition of at least two serious problems for the structuralist.

First, note that irrespective of the circumstances, or the values put into, say, Newton's inverse-square equation, Newtonian space and time are wholly unaffected; as above, they posses an immutable, invariant, absolute structure. By contrast, elaborating on the connection just noted, crucially, solutions to the Einstein equation determine the description of how spacetime itself is structured; spacetime itself can be contorted and twisted, so structured, depending on the values put into the equation and the auxiliary hypotheses to which the equation is conjoined. When contrasted against Newtonian immutable space and immutable time, utterly indifferent to the values put into Newtonian formulae, the inextricable connection between the Einstein equation and spacetime structure further illuminates just how fundamental and dramatic is relativity's structural upheaval.

The second major problem for the structuralist: depending on the specifics of the solution, the Einstein equation can be used to express innumerably many structured spacetimes-from those consisting of a few massive objects to those with hundreds of billions of galaxies made up of hundreds of billions of stars; from those that are static to those that are expanding and even those accelerating in their expansion; from those with only visible matter to those with dark matter and dark energy, and (to the surprise of Einstein, initially) those that have no modicum of matter but are nonetheless in motion. The Einstein equation can accommodate all such worlds, all such spacetime structures, and more. The very significant implication: despite the apparent promise the Einstein equation has for constituting structure, especially given its direct connection to spacetime structure, we cannot equate it with, or even claim it presents to us (using Steven French's apt phrase) the structure of our world. Were it to exhaust one's beliefs about macrostructure, we would have little if anything to justifiably believe. It is structurally informative only when it is given values; the structure it offers is contingent on those values, and, it would at least appear, Einstein's equation tells us only how the world would be structured if the equation were given values. Finding solutions to that equation and auxiliaries pertaining to, for instance, matter is a precondition for forging even a minimal connection between that equation and our world of experience.

Because Einstein's equation allows for so many solutions and permits so many worlds, it turns out to be incredibly difficult to find values and appropriate sets of auxiliaries that will connect the equation to any kind of actual experiences in this world. Due to its extraordinary permissibility, not only is the Einstein equation infamously difficult to "solve," the way and extent to which the solutions that have been found actually relate to our world is often extremely ambiguous. For instance, whether Einstein's equation genuinely implied the existence of black holes was debated for

\footnotetext{
${ }^{3}$ In accord with this point, I break from standard practice here and reference "Einstein's equation" in the singular.
}

decades, both Einstein and Eddington denying that it did. Similarly disputed were its implications for the existence of gravitational waves. Eddington quipped that "the only speed of propagation relevant to"-at least two out of three kinds of-gravitational waves is "the speed of thought" $(1922,269)$. To the considerable surprise of most physicists (and the media) exactly one century later, in a February 1916 letter to Schwarzschild, Einstein wrote, "there are no gravitational waves analogous to light waves" (1916a, 196). And although Einstein soon argued for the existence of gravitational waves, as early his (1916b) and (1918), he and Rosen wrote a paper in 1936 in which he again denied their existence. (On the latter, see Kennefick (2005)). Illustrating the difficulty of solving the equation, due to its lack of informative content: even when gravitational waves were accepted as a genuine possibility, solving the equation for physical situations that would render them detectable proved extraordinarily trying. ${ }^{4}$ Warnings about the near impossibility of doing so trace at least as far back as 1957, at the Chapel Hill Conference. Charles Misner suggested one would need "a computing machine better than anything we have now, and many programmers and a lot of money" and that without the proper specification of precise "constraints," "either the programmer will shoot himself or the machine will blow up" (1957, 87). A half-a-century of effort and millions of dollars were put into deploying computers and then supercomputers to solve-that is, conjure up the auxiliaries hypotheses-for a situation in which two black holes orbit one another and then collide, thereby producing gravitational waves. Conference attendees Robert Lindquist in 1964 and Bryce Dewitt in the 1970s were among those who struggled for such a solution. However, results continued to fall under what essentially amounts to Misner's second option. A sub-branch of relativistic physics, numerical relativity, arose during the decades of such efforts, and, finally, Frans Pretorious, after months with a supercomputer, and the courage, or audacity, to invoke a set of unrealistically simple auxiliary hypotheses finally solved the problem in his (2005), 90 years after Einstein had presented his general theory. Following decades of claims that gravitational waves had been detected (and a short time prior to this article going to proofs), physicists announced with a clear consensus-in a paper with over one thousand authors-that gravitational waves had been "observed" by the LIGO detectors on September 14, 2015: "We report the first direct detection of gravitational waves and the first direct observation of a binary black hole system merging to form a single black hole" (B. P. Abbott et al., 2016, 061102-1).

This difficulty in deriving empirically informative predictions is a symptom of the fact that the Einstein equation permits so many different worlds, and it helps us to see that, by itself, Einstein's equation cannot be equated with any kind of informative structure, of spacetime or anything else. By way of values that can be inserted, and auxiliaries to which the equation can be conjoined-including those that invoke, say, invisible matter and invisible energy - the equation, again, permits innumerably many spacetime structures. As above, because the equation relates matter to spacetime structure, it does have implications for structure once auxiliaries pertaining to, for instance, matter are articulated; however, because it allows for any situation regarding matter, there is no particular structure that the equation implies. Expressed in the context of predictive success, so central to any warrant for structuralism, Einstein's equation by itself fails to render likely, let alone, entail, a single temporally-novel or use-novel prediction about empirical phenomena. In fact, by itself, it tells us precisely nothing about

\footnotetext{
${ }^{4}$ And this is so even bracketing the related challenge of developing an apparatus - such as the Laser Interferometer Gravitational-Wave Observatory (LIGO) - that would allow one to detect them.
} 
anything we can observe. (In terms I will return to below, to believe the Ramsey sentence of, or percepts entailed by, the Einstein equation is to believe nothing at all, since, by itself, that equation has no Ramsey sentence and implies precisely no percepts.) Above I suggested that, in our attempt to identify a structuralist commitment, Einstein's equation itself appears to hold promise. (And given the significance of general relativity, structuralists are compelled to address it some way or another.) However, we now see that, were we to equate Einstein's equation with structure, the structure to which we would commit ourselves becomes wholly unspecifiable, and so utterly vacuous.

Although structuralism is taken to hold considerable promise as an answer to the historical challenge to scientific realism, all of this bears significantly on the mounting threat I am continuing to unfold, the threat of the explanatory vacuity of "structure." And one reason I stress these particular points about Einstein's equation is to suggest that this perceived promise may often trade on the vacuity of mathematical equations, Einstein's being a particularly interesting and illustrative example. It is not the only example, of course. An inextricable connection to spacetime is not required. Consider the posit of a trans-Uranian planet that led to identifying a point of light in the sky. It is one thing to claim that planetary posit to be purely structural; however, it is quite another challenge to claim that Leverrier and Adams had the right structure. The posits deployed pertaining to, not only the mean distance and the mass, but also the eccentricity, orbital period, longitude of Neptune's perihelion, etc. were patently, and structurally, false. Similarly, adding to our cases above, although Lagrange and Leverrier made predictions about Venus that were in close accord, they did so by deploying dramatically divergent constituents regarding Venus's orbit and mass. To claim victory over deployment realism on such challenges, the structuralist will be tempted to exploit the free Newtonian variables of mass and distance, each of which can be adjusted, provided the other is adjusted, embracing the fact that the relation between the variables nonetheless obtains. However, discarding any specifics pertaining to mass and distance, no nonarbitrary restriction on those values remains; in that case, to exploit that relation, by itself, is to fall straight into the kind of vacuity suggested here. Moreover, even that degree of permissibility may not suffice for a historical retention of structure: since, according to special relativity, any number of variations between mass and distance can obtain depending on the reference frame-including for instance, the reference frame of the light we are observing, over which our favored reference frame, explicitly, has no greater privilege - it is wholly unclear that even special relativity retains that Newtonian mass-distance structural relation, requiring an even greater depletion of content in the notions of "relation" and "structure."

\subsection{Depleting the content of "structure"}

On that theme, returning now to our historical case studies, wanting to claim that novel predictions result from obtaining the right structure, our structuralist has no choice but to make the notion of structure extremely open, allowing, for instance, that pellets of mass structurally approximate massless waves, that an inverse relation structurally approximates an inverse-square relation, that "the velocity of light changes" structurally approximates "the speed of light is constant," that "the velocity of light pellets can be superseded" approximates "nothing can travel faster than $c$," etc. The slippery notion of "structure" (and the allure of the "abstract") may not prohibit any of this; however, crucially, the greater ease with which such historical alleviations are purchased, the more depleted and uninformative is structuralism's content. Notably, in discussing the structure of light, we have not yet added Fresnel's theory into the mix. Forced by novel successes to concede that Kepler, Michell, Laplace, and von Soldner got the structure of light correct, and that Fresnel also got that structure correct, will render empty the achievement of getting the structure of light correct. We are seeing a mounting threat posed by the historical counterinstances: in want of accommodating them the structuralist is forced toward an increasingly loose notion of structural approximation, one that, with each stretch, threatens to commit structuralism to an extra-ordinary depletion of the logical content of science itself: if science gives us only such structure, it gives us extraordinarily little, if anything at all. ${ }^{5}$ Emphasizing the absolute importance of structure, the structuralist is committed to claiming that, when an instance of theorizing-for instance, denying the variance of light, denying the instantaneous force of gravitation-makes all the difference in the attempt to fit our theorizing to the data and to make novel predictions, that impacting difference will be a structural one. Moreover, again emphasizing the absolute importance of structure, the resulting difference will generally be structural. However, in each case where we had novel success we must attribute that to the fact that we had the right structure; and where, after such novel success obtains, a structural difference is introduced because it makes all the difference to other phenomena and novel predictions, the structuralist is committed to our having the right structure not only after but crucially before that posited structural difference. This is bound to drain "structure" of its content-and, so, drain it of explanatory strength: the structuralists exploitation of the now increasingly apparent flexibility of "structure" decreases the structuralist's ability to claim that "getting the structure right" will do anything to render novel predictive successes "a matter of course," to use Peirce's phrase, or even likely. Taken by itself, this mode of criticism is distinct from both the historical and underdetermination arguments-but can be traced back as a challenge to scientific realism through Lyons (2006), (2003), to Laudan (1981). ${ }^{6}$ Here, however, I am using the above historical challenges to leverage the point and to direct this problem of explanatory vacuity against structuralism.

\section{Structuralism and its most recent restrictions}

To remedy this depletion of "structure" and rein in that threat of explanatory vacuity, the structuralist is forced to impose severe restrictions on structure. And structuralists have introduced restrictions. ${ }^{7}$ Most notably, perhaps, needing to give greater content to his original (1989) hint at structuralism, Worrall's current structuralism is committed to, and only to, a theory's Ramsey sentence (Worrall \& Zahar, 2001), (Worrall, 2007; 2011). This turn to the Ramseyfication of theoretical content is itself a dramatic shift for his structuralism, and we would be remiss to neglect it here. From considerations above, I will direct a series of challenges against this dramatic shift. First, as we briefly noted above, Einstein's equation, by itself, contains exactly zero observational content; since structuralist Ramseyfications require observational

\footnotetext{
${ }^{5}$ Or, one might say, since the structuralist account of science renders the content of science exactly as vacuous as the structuralist's conception of "structure," both are now rendered vacuous indeed.

${ }^{6}$ In fact, against the standard scientific realist, Worrall himself discusses the difficulty before putting it "into abeyance" $(146,1989)$. See footnote 7 . And although Psillos, a deployment realist, fails to address this as a problem for approximate truth (Lyons, 2006, 556), he nonetheless appropriately invokes such a point as a challenge to structuralism in his (1999, 153-4). Albeit, like Laudan, niether invokes historical counter-instances for leverage as I am doing here.

${ }^{7}$ It may well be that, up until now, the reader familiar with these restrictions has expected that they would solve the problems I've raised. In this section I hope to relieve the reader of that expectation.
} 
content, to believe a structuralist's Ramseyfied version of Einstein's equation by itself would be to believe exactly nothing. Or if we want to emphasize "percepts," the set of percepts that Einstein's equation entails is unequivocally empty. (So is the set from which one can make an "upward" inference to that equation.) Now Worrall appropriately recognizes the need for a system (2011), which we realize will often include numerous auxiliary hypotheses, so avoids commitment to the non-existent Ramsey sentence of Einstein's equation by itself. Nonetheless, second, theoretical systems themselves are continually evolving, and since a belief is a belief at a specific time, there must be a specific time at which we are justified in believing the system's Ramsey sentence, and we are left wondering just what time this would be. Were Einstein or Eddington structuralists, one must wonder at which stage in the evolution of the relativistic system would they have properly committed themselves to its Ramsey sentence. Worrall's own shift to Ramsey sentences (Worrall \& Zahar, 2001) takes place before, say, Pretorious's (2005) solution for gravitational waves as well as the LIGO confirmation (Abbott et al., 2016), each of which rendered for the system a new Ramsey sentence, as I will note below. And third, rather than repeat each case detailed above, I'll simply point out the following: among the entire collection of theoretical systems of constituents responsible for the successes detailed in this paper, there is not one whose Ramsey sentence is not patently false by present lights. In no such instance can the truth of the system's Ramsey sentence explain that system's success. The divergent poles of, on one end, explanatory vacuity, and, on the other, a failure to account for counterinstances lead to the same result, an inability to explain success.

Fourth, although general relativity and quantum field theory (discussed by Worrall in 2011) appear to be our best contemporary theories, their conjunction is responsible for what appears to be the most dramatic - and in the realism debate, as far as I know, wholly unacknowledged-discrepancy between prediction and data in the history of science: the predicted value of $\lambda$ diverges from the data by up to 120 orders of magnitude. (See, for instance, Frieman, Turner, and Huterer (2008, 20); Ellis, Elst, Murugan, and Uzan (2011, 1); Murugan, Weltman, and Ellis (2012, 2-3).) That given, our structuralist cannot even appeal to the Ramsey sentence of the conjunction of our very best theories; even that Ramsey sentence is-surprisingly, to many-rendered patently false by the data. Fifth, important to the comparative evaluation in this paper, the fourth point leads us to another surprising result: the deployment realist, who makes no commitment to the truth of a deployed system's Ramsey sentence, may well fare better with regard to contemporary science than our Ramsey-sentence structuralist.

Sixth, although Worrall does discuss "in the limit" preservations with regard to mathematical equations, this does not automatically carry over to, say, truth-in-the-limit of the Ramsey sentence: for instance, in that earlier text he acknowledges that the observation statements derived from the Newtonian system fail to be, strictly speaking, true (1989, 143, footnote 6). And insofar as "true" in the limit requires truth in the specific observation statements, that system's Ramsey sentence cannot be, strictly speaking, true, even in the limit. Seventh, this example also tells us that, although successful predictions themselves may be true, even those extremely limited "portions" of the Ramsey sentence that express the successful predictions need not be true, and that, in fact, we cannot insist that they are. (On this, see Lyons, 2003, 893-4.) Eighth, mindful of these last points as well as the second point, regarding the evolution of systems, and considering for instance, the details of, say, the "Eddington experiment" or the claims of LIGO's successful detection, there are numerous auxiliaries that are articulated after a test of a novel prediction occurs. For instance, as we all realize, the confirming observation claims relevant to LIGO depend on other theoretical claims-for instance, the claim that no interfering factors affected the laser interferometers-which in turn depend on yet other observation claims, presumably also now part of the Ramsey sentence. That given, the system's Ramsey sentence that is directly relevant to the novel prediction is not even articulated until after the test of the prediction is completed (or, the confirmation of the prediction involves changing the system's Ramsey sentence). This is not to say that a not-yet-articulated Ramsey sentence cannot be true; the point is rather that it cannot be believed.

Because "true-in-the-limit" is bound to receive plenty of play in the realist's mind, as a ninth challenge to Worrall, we can pause here to note that, not only Worrall, but realists in general, would be mistaken to depend on "in-the-limit" qualifications. A quick point regarding applicability (see Section 1): in-the-limit is a comparative notion, with a later theory specifying just what that limit is; but in absence of the future theory we cannot, of course, use it to articulate a present commitment. Approaching this from another direction, consider a new theoretical system, prior to any evidence for it, along with the assertion that it is (say, structurally) true-in-the-limit. Because "in-the-limit" is itself unrestricted, a statement can be ascribed in-the-limit properties even though that statement is patently (structurally) false. For instance, taking the limit as the edge of a black hole, it can be, by contemporary lights, true-in-the-limit "that there is no inverse square relation to be had, that the universe is profoundly non-Euclidean, that gravitation and acceleration are inequivelent, etc." Likewise, and more simply, "the earth is flat," "the entire universe is the surface of a two-dimensional table" are, by contemporary lights, true-inthe-empirical-limit, if not true-in-the-limit. However clearly, each of these is also, by contemporary lights, patently false. With no limits to, i.e., no restrictions imposed on, what can be designated the limit, the qualifications "true-in-the-limit," "true-in-thestructural-limit," "the-right-structure-in-the-limit," or even "true-in-the-empirical-limit" are bereft of informative content. Although, in the face of the historical threat, such clichés may offer momentary psychological comfort to realists, resorting to such clichés sacrifices the explanatory argument on which the realist justification depends: in the end, falling back on such ad hocery the structuralist claim need mean little, if anything, more than the system is empirically successful where it is empirically successful.

Tenth, returning more specifically to a structuralist commitment to a system's Ramsey sentence, Worrall himself rejects the realist's appeal to approximate truth (1989). In a thorough discussion of the history of appeals to the Ramsey sentence, among the many significant challenges Simon Saunders and Kerry McKenzie (2014) raise, but one (e.g. 149) is that it remains unclear just how a past system's Ramsey sentence would approximate that of another system. Mark Newman, discussing a Lewisian conception of Ramsey sentences, argues that the false posits pertaining to "mass" will permeate the Newtonian system's Ramsey sentence $(2005,1383)$. To Newman's point, and in the spirit of Saunders and McKenzie, I would add that this may well hold for Worrall's "original, rather than Lewisian" (Worrall, 2007, 149) Ramsey sentence; Worrall writes, "the theoretical terms surely live on within the Ramsey sentence via the structure that they impose on the observational content. (That is, after all, what structural realism is about.)" (2007, 152). And a number of the false deployed theoretical posits identified above will likewise pollute the terms included in the Ramsey 
sentence, bringing their falsity with them. Consider for instance, the "observation term," "light." If Worrall does embrace a move to the approximate truth of a system's Ramsey sentence, it will surely require work, especially given Worrall's own doubts about approximation $(1989,156)^{8}$ and the fact that Worrall himself insists that the only acceptable or even possible theory of reference is "global descriptivism" $(2011,169)$. Eleventh, although recognizing that his position borders on antirealism, Worrall contends that much of what is included in the Ramsey sentence is theoretical. However, given the collective force of points made in this paper, it is unclear whether, upon discarding all the falsity identified above, anything would remain to distinguish Worrall's position from antirealism. Finally, in conjunction with the third point above, in the off chance that Worrall can somehow provide an account of Ramsey sentence approximation that evades the many challenges raised here (and in the papers just cited), he will be pressed to show the deployment realist just why his notion of approximation cannot equally be invoked to save the realist's theoretical constituents, threatening the primary motivation for his structuralism. ${ }^{9}$

Although my focus has been on epistemic structuralism, for instance, the variants embraced by Worrall, I expect that my historical survey has serious implications for other forms of structuralism, such as Ladyman's (and possibly French's) ontic variant. Ladyman appropriately rejects appeal to a system's Ramsey sentence $(2011,100)$ and, beyond the empirical assertions, ${ }^{10}$ he appeals to the system's modal content, to its claims regarding, for instance, physical necessity. Not only is this another set of restrictions structuralists have introduced, it is that which distinguishes Ladyman's structuralism from anti-realism, e.g. that of van Fraassen (Ladyman, 1998, 2000, 2011): denying such commitments, antirealism lacks the explanatory strength needed to make sense of science (Ladyman, 1998, 416-7). This modal structuralism, involving, for instance, commitment to a system's assertions regarding physical necessity, may well hold promise for addressing the explanatory vacuity problem. Ontic though this structuralism may be, it is, like its epistemic counterpart, also motivated by a desire to address the historical challenge (Ladyman, 1998, 2000, 2011). The posit that nothing can travel faster than $c$, is, for Ladyman, the sine qua non of physical necessity, and more generally, an example of the kind of modal claim that our contemporary system entails and to which the structuralist is committed. However, considerations above push us to acknowledge that modalities such as physical necessity are system dependent. And, crucially, we have seen that, within the Newtonian system, the speed of light varies, while the action-at-a-distance gravitational force is invariantly instantaneous. Moreover, for Michell and von Soldner, it is that invariantly instantaneous gravitational force that overcomes the speed of light corpuscles in order to slow them down. Hence, a brazen denial of the current posit that nothing can travel faster than the speed of light is directly responsible for successful novel predictions we have traced from the Newtonian system. (Add to this, other points from Section 3.2 on the dramatic structural clash between the Newtonian system and the contemporary conception of

\footnotetext{
${ }^{8}$ Worrall notes, "Every false theory, of course, has infinitely many false consequences (as well as infinitely many true ones)" and a theory that is close to the truth gets some things "totally wrong" $(1989,145)$.

${ }^{9}$ I suggest that, if he could provide some account of approximation, and if it is of the right kind, the present paper would afford both a context and fuel for separating his approximation from the deployment realist's; but these are big "ifs," and until they are achieved, they do nothing to alleviate the many concerns raised here for Worrall's position.

10
}

For our purposes, we can allow this to be the very non-demanding claim that, say, empirically successful predictions are approximately true. the relations between the invariance of $c$ and spacetime, mass, energy, and distance.). Our current theoretical system similarly renders physically impossible a number of the deployed constituents we have identified above, e.g, that the constituents deployed in Newton's (1684) system obtain. The deployed systems we have examined can and do, rather easily but no less dramatically, patently defy the modal content of our contemporary theoretical system. Nor does it appear that modal commitments permit the kind of approximation or in-the-limit maneuvers we challenged above.

\section{Conclusion}

In order to comparatively evaluate deployment realism and structuralism, this paper has introduced a new set of historical case studies. Needing to justify belief in their respective commitments both deployment realists and structuralists depend on the nomiracles argument. However, the explanatory demands of that argument, combined with a careful look at the history of science, reveal a serious tension between the commitments of, not only the deployment realist, but also the structuralist. My claim is that, with threatening historical data in hand, an inverse relation obtains between the structuralist's capacity for accommodating those data, on the one hand, and the capacity to bear on, so explain, novel predictive success, on the other; the specific historical challenges introduced here reveal that securing one of these requirements for a tenable position comes at the expense of the other. In terms of the vacuity of equations, discussed above: their uninformative nature, along with the structuralist's explanatory demand, forces the concession that particular values must be put into the equations. Yet, we've seen, the better structuralism fares in specificity and explanatory power, the worse it fares against history; and the better structuralism fares against history, in terms of retention, the worse it fares in content and explanatory power. Whether it be due to a concession to miracles or to the elimination of content and depletion of explanatory power, neither deployment realism nor structuralism can explain the series of novel predictive successes we've discussed, some of the most important successes in the history of science. Either way, like deployment realism, structuralism, at least in the forms discussed here, is significantly threatened by the very historical challenge it was introduced to answer.

\section{Acknowledgements}

This paper was first presented on 15 June 2014 at Case Western Reserve University. Among those to whom I am indebted for relevant conversations and correspondence are Horia Petrache, Adam Hayden, and our Indiana-Purdue Philosophy and Physics Group; Ioan Muntean, Kerry McKenzie, Chris Haufe, Peter Vickers, Steven French, James Ladyman, Ioannis Votsis. Research for this article was supported by the Arts and Humanities Research Council, United Kingdom AH/L011646/1.

\section{References}

Abbott, B. P., Abbott, R., Abbott, T. D., Abernathy, M. R., Acernese, F., \& Ackley, K. (2016). Observation of gravitational waves from a binary black hole merger Physical Review Letters, 116, 061102.

Campbell, W. (1909). The closing of a famous astronomical problem. The Popular Science Monthly, 494-503.

Campbell, W. (1919). The meeting of the royal astronomical society, Fowler, A. chair July 11, 1919. The Observatory, 42, 297.

Earman, J., \& Glymour, C. (1980). Relativity and eclipses: The British eclipse expeditions of 1919 and their predecessors. Historical Studies in the Physical Sciences, 11(No. 1), 49-85.

Eddington, A. (1922). The propagation of gravitational waves. Proceedings of the Royal Society of London, Series A, 102, 268-282. 
Einstein, A. (1916a). Doc. 194. To Karl Schwarzschild. Princeton: Princeton University Press. The collected papers of Albert Einstein, Volume 8: The Berlin years: Correspondence, 1914-1918 (English translation supplement) Translated by Ann M. Hentschel.

Einstein, A. (1916b). Approximative integration of the field equations of gravitation (pp. 201-210). The collected papers of Albert Einstein, Volume 6: The Berlin years: Writings, 1914-1917. (English translation supplement) Translated by Alfred Engel.

Einstein, A. (1916c). Relativity: The special and general theory (3rd ed.). New York: Henry Holt and Company (1920), Translated by Robert W. Lawson.

Einstein, A. (1918). On gravitational waves (pp. 9-27). The collected papers of Albert Einstein, Volume 7: The Berlin years: Writings, 1918-1921 (English translation supplement) Translated by Alfred Engel.

Einstein, A. (1936). Do gravitational waves exist?. unpublished manuscript, submitted to, but rejected by Physical Review.

Ellis, G., Elst, H., Murugan, J., \& Uzan, J. (2011). On the trace-free Einstein equations as a viable alternative to general relativity. Classical and Quantum Gravity, 28 225007-225017.

Frieman, J., Turner, M., \& Huterer, D. (2008). Dark energy and the accelerating universe. Annual Review of Astronomy and Astrophysics, 46, 385-432.

Glendenning, N. K. (2007). Special and general theory of relativity: With applications to white dwarfs, neutron stars and black holes. New York: Springer.

Grünbaum, A. (1976). Ad hoc auxiliary hypotheses and falsificationism. The British Journal for the Philosophy of Science, 27, 329-362. No. 4 (Dec., 1976)

Herschel, J. (1849). Outlines of astronomy, 1869 (10th ed.). London: P. F. Collier and Son.

Jaki, S. (1978). Johann Georg von Soldner and the gravitational bending of light with an English translation of his essay on it published in 1801. Foundations of Physics, 8, 927-950.

Kennefick, D. (2005). Einstein versus the physical review. Physics Today, 58(9), 4348.

Kepler, J. (1596). Mysterium Cosmographicum, the secret of the universe, 1621 (2nd ed.). New York: Abaris Book. translated by A. M. Duncan, 1981.

Kepler, J. (1609). New astronomy. Cambridge: Cambridge University Press. translated by W. H. Donahue, 1992.

Ladyman, J. (1998). What is structural realism? Studies in History and Philosophy of Science, 29(3), 409-424.

Ladyman, J. (2000). What's really wrong with constructive empiricism? Van Fraassen and the metaphysics of modality. British Journal for the Philosophy of Science, 51, 837-856.

Ladyman, J. (2011). Structural realism versus standard scientific realism: The case of phlogiston and dephlogisticated air. Synthese, 180, 87-101.

Laudan, L. (1981). A confutation of convergent realism. Philosophy of Science, 48, 19-

Lyons, T. (2003). Explaining the success of a scientific theory. Philosophy of Science, $70,891-901$.

Lyons, T. (2006). Scientific realism and the Strategema de Divide et Impera. The British Journal for the Philosophy of Science, 57, 537-560.

Michell, J. (1784). On the means of discovering the distance, magnitude, \&c. of the fixed stars, in consequence of the diminution of the velocity of their light.... In
In a letter to Henry Cavendish, philosophical transactions of the Royal Society of London (Vol. 74, pp. 35-57).

Misner, C. (1957). Solving the initial value problem using cartan calculus. In C. M. DeWitt, \& D. Rickles (Eds.), The role of gravitation in physics, report from the 1957 Chapel Hill conference. Max Planck Research Library for the History and Development of Knowledge, Volume 5 of Sources, Berlin, 2011. Edition Open Access.

Murugan, J., Weltman, A., \& Ellis, G. (2012). The problem with quantum gravity. In J. Murugan, A. Weltman, \& G. F. R. Ellis (Eds.), Foundations of space and time: Reflections on quantum gravity (pp. 1-7). New York: Cambridge UP.

Newcomb, S. (1910-1911) (11th ed.). "Mercury," encyclopedia britannica (11th ed.), (Vol. 18) p. 155, 1910-1911.

Newman, M. (2005). Ramsey sentence realism as an answer to the pessimistic meta-Induction. Philosophy of Science, 72, 1373-1384.

Newton, I. (1684). De motu corporum in gyrum. In D. T. Whiteside (Ed.), The preliminary manuscripts for Isaac Newton's 1687 Principia, 1684-1686 (facsimiles), 1989. Cambridge: Cambridge University Press. Translated in (2008) The Mathematical Papers of Isaac Newton: Volume 6.

Newton, I. (1687). Sir Isaac Newton's mathematical principles of natural philosophy and his system of the world (3rd ed.) Translated Andrew Motte (Facsimile Reprint) Kessinger Publishing, LLC (January 24, 2003)

Newton, I. (1704). "Book III," opticks: Or a treatise of the reflexions, refractions, inflections and colours of light. Also two treatises of the species and magnitude of curvilinear figures. London: Sam. Smith and Benj (Walford, printers to the Royal Society)

Pretorius, F. (2005). Evolution of binary back hole spacetimes. Physical Review Letters, 95(12), 121101.

Psillos, S. (1999). Scientific realism: How science tracks truth. London: Routledge.

Saunders, S., \& McKenzie, K. (2015). "Structure and logic," physical theory: Method and interpretation (pp. 127-162). Oxford: Oxford University Press.

von Seeliger, H. (1896). Die scheinbare Vergrösserung des Erdschattens bei Mondfi nsternissen (pp. 383-448). Abhandlungen der mathematisch-physikalischen Classe der königlich bayerischen Akademie der Wissenschaften 19.

von Soldner, J. G. (1801). On the deviation of a light ray from its motion along a straight line through the attraction of a celestial body which it passes close by. In Jaki, S. (1978) "Johann Georg von Soldner and the gravitational bending of light, with an English translation of his essay on it published in 1801," Foundations of Physics, 8 pp. 927-950).

Worrall, J. (1989). Structural realism: The best of both worlds? In D. Papineau (Ed.), 1996, philosophy of science (pp. 139-165) Oxford: Oxford University Press.

Worrall, J. (2007). Miracles and models: Why reports of the death of structural realism may be exaggerated. Royal Institute of Philosophy Supplement, 82(61), $125-154$.

Worrall, J. (2011). Underdetermination, realism and empirical equivalence. Synthese, $180,157-172$.

Worrall, J., \& Zahar, E. (2001). Appendix IV: Ramseyfication and structural realism. In E. Zahar (Ed.), Poincaré's philosophy: From conventionalism to phenomenology (pp. 236-251). Chicago: Open Court. 